UDC 616.12-005.4-071

DOI https://doi.org/10.31718/mep.2021.25.1-2.05

\title{
ENGLISH VERSION: EVALUATION OF ELECTRO-PHOTONIC EMISSION ANALYSIS INDICATORS IN PATIENTS WITH NON-COMMUNICABLE DISEASES - CORONARY HEART DISEASE*
}

Nevoit G.V.

Ukrainian Medical Stomatological Academy, Poltava, Ukraine

This study is a part of the initiative research project "Development of algorithms and technology for introducing a healthy lifestyle in patients with non-communicable diseases based on the study of psycho-emotional status" (State registration No. 0116U007798, UDC 613:616-052:159.942:616-03).

A clearer fundamental knowledge of the principles of the electromagnetic organization of the human body can provide a scientific basis for further solving a number of modern medical problems, and this can help overcome the global pandemic of chronic non-communicable diseases The aim of the study is to improve methods of diagnosis, treatment and prevention of chronic non-communicable diseases by studying and using the latest science-intensive technologies. Electro-photonic emission analysis was conducted on 126 patients with coronary heart disease. The obtained results were compared with the control group - 56 functionally healthy individuals. The electro-photonic emission analysis from human fingers is proposed by the author as one of the promising methods for possible study of systemic electromagnetic energy processes of the human body in the implementation of objective structured clinical examination.

Key words: non-communicable diseases, coronary heart disease, objective structured clinical examination, photonic emission.

\section{Introduction}

The scientific substantiation of the electromagnetic essence of the basis of all metabolic processes in living biological systems has deepened the fundamental knowledge about the structure and functioning of the human body. This necessitated further study of electromagnetic phenomena of the human body to improve the methodology of their clinical and diagnostic interpretation. A clearer fundamental knowledge of the principles of the electromagnetic organization of the human body can provide a scientific basis for further solving a number of modern medical problems, and this can help overcome the global pandemic of chronic non-communicable diseases (NCDs). This can be done by improving the diagnosis and prevention of NCDs through the introduction into modern medical practice of modern scienceintensive technologies, integration of systemic fundamental physico-biological knowledge and changing the existing electrochemical paradigm of metabolism to magnetoelectrochemical [7].

Ultra-weak photon emission (UPE) is one of the most promising for studying the phenomena of living biological systems, including humans. UPE reflects the systemic energy processes of the micro-level of functioning of tissues of the human body. It is proved that UPE occurs due to the emission of photons by living molecules and human tissues during metabolic reactions. Excessive oxidative metabolism is one of the decisive factors in NCDs. Therefore, the study of photon emission can be considered as a potential tool for assessing the functioning of tissues and organs to reflect the oxidative processes at the cellular level. Today, the spectrum and intensity of UPE are considered to be recognized, and the main source, statistical distribution, fractality are partially understood. UPE registration requires ultra-sensitive digital equipment and is technically complex. The UPE regis- tration process can be simplified by additional electromagnetic stimulation of tissue radiation. Thus, a special electromagnetic effect on the skin of the human finger creates ionization of gas molecules around it due to emitted electrons and photons, charges the skin surface and this causes the transport of electrons from the human skin surface through structural complexes of biomolecules into the pulsed current circuit.

The method of Electro-Photonic Emission Analysis (EPEA) was developed on this principle and the certified hardware and software complex Bio-Well (Russia-USA) was created. EPEA of human fingers using the Bio-Well device is proposed by us as one of the promising methods of possible study of systemic electromagnetic energy processes of the human body in the objective structured clinical examination of patients, including patients with NCDs $[2-4,7]$.

The aim of the study is to evaluate the clinical and diagnostic capabilities of EPEA in reflecting the systemic information energy processes of the human body to increase the effectiveness of measures to prevent and treat NCDs by improving their diagnosis and prevention through the introduction of modern science-intensive technologies and scientific concepts of magnetoelectrochemical metabolism.

\section{Materials and methods}

Open, non-randomized, controlled study was performed on the basis of the educational and practical center of biophotonics and valeology of the Department of Internal Medicine and Emergency Medicine of the Educational and Scientific Institute of Postgraduate Education at Ukrainian Medical Stomatological Academy (UMSA) and physiotherapy department of the municipal enterprise "Poltava M.V. Sklifosovsky Regional Clinical Hospital of the Poltava regional council".

The EPEA study of NCDs patients was a fragment of

*To cite this English version: Nevoit G.V. Evaluation of electro-photonic emission analysis indicators in patients with non-communicable diseases - coronary heart disease // The Medical and ecological problems. - 2021. - Vol 25, № 1-2. - P. 19-21. 
it. A complete EPEA of ten fingertips was performed in 68 patients with coronary heart disease: angina pectoris (median age are $60(45 ; 87)$ years, $36(59 \%)$ are men; it is group 1) and 58 patients with coronary heart disease: myocardial infarction in the subacute stage (median age are $64.5(43 ; 80)$ years, $45(78 \%)$ are men; it is group 2). All patients had a verified diagnosis, comorbid pathology of the internal organs and were treated at the municipal enterprise "Poltava Regional Clinical Medical Cardiovascular Center of the Poltava Regional Council". 56 functionally healthy respondents (students, interns, clinical residents of UMSA) who do not regularly engage in sports formed the Control Group (median age are 23 (19; $35)$ years, $18(32 \%)$ are men. EPEA was performed twice during the implementation of the author's comprehensive method of functional research. It included performing valeological testing, a brief recording of heart rate variability (HRV) with an orthostatic test, and ATM express testing.

EPEA was performed before HRV background recording and after orthostatic testing. EPEA was performed on a digital device Bio-Well 2.0 (Russia-USA). Bio-Well 2.0 is a certified measuring device for use in a wide range of scientific and practical research. Bio-Well 2.0 is registered in 70 countries and has CE, EU and FDA certificates. The method of registration of the device is based on the physical phenomenon of electrophoton radiation. It is the flow of electrons from a conducting object under the influence of a high-frequency electromagnetic pulse of high intensity, which creates photon radiation (glow) in the gas air. This is a gas discharge visualization (GDV). The Electro-Photonic Imaging (EPI) digital software package is used with the Bio-Well GDV Camera 2.0. It provides the possibility of digital photoregistration of the parameters of the phenomenon of electrophone radiation from human fingers, transfer to a virtual database, processing and analysis of results. The EPI software package is based on computer analysis of images of photons emitted by a subject in a strong pulsed electromagnetic field. The full scan mode was used in this fragment of the study. Full scan is a ten-finger Bio-Well sequential photo recording mode. It allows you to get indicators of the functional (energy) state of man on the basis of analysis of the intensity of the glow and its geometric parameters. The device is based on the theoretical basis and methods of registration and analysis. This methodology has been calculated gradually over 20 years of clinical trials of various device modifications [1, 4-6, 7].

Direct and calculated parameters were evaluated by us: the Area (S, number of pixels of the Glow Image (GI); its ratio of Area of the finger glow to the Area of glow of calibration cylinder (for a sector or the whole image); the Normalized Area is the ratio of GI Area to the area of the inner oval; the Intensity is the average intensity of all the pixels from the $\mathrm{Gl}$; the Energy of Glow (E, Joules/J; its integral indicator of functional state; its a numerical estimate of the glow energy of the photographed emission of photons multiplied by the Area, Intensity and the correction factor); the Stress (c.u.; its an integral parameter of the functional state of a person, its a numerical assessment of the psychoemotional and functional state of the examined person, based on the assessment of the curvature of the outer contour of the photon emission of the fingers); the Form Coefficient (FC; its calculated according to the formula: $F C=L 2 / S$, where $L$ is the length of the Gl external contour and $S$ is the GI Area; it reflects the degree of roughness of the outer contour of the photon emission image. it is assessed for each finger of the hand, for fingers on both hands and separately for the right and left hands in average parameters;); the Entropy Coefficient (EC; its the ratio of outer contour to the inner contour lengths); the Balance (B, \%), Balance left (BL,\%) and Balance right $(\mathrm{BR}, \%)$ reflect the presence or absence of asymmetry (the phenomenon of lateralization); the Energy Field (EF) is an abstract parameter, it is the result of a computer simulation of the glow of ten fingers; left and right Energy Field Energy $\left(\mathrm{E}_{\mathrm{EF}} \mathrm{L}, \mathrm{E}_{\mathrm{EF}} \mathrm{R}, \mathrm{J}\right)$; left and right Energy Field Area $\left(\mathrm{S}_{\mathrm{EF}} \mathrm{R}, \mathrm{S}_{\mathrm{EF}} \mathrm{R}, \mathrm{c} . \mathrm{u}\right.$.).

Statistical analysis was performed using the Prism 5.0 software package. The data obtained are presented as mean values with their mean error $(\mathrm{M} \pm \mathrm{m})$. The MannWhitney test was used to determine the statistical significance of differences between groups. Differences were considered significant at $p<0.05$.

\section{Results and discussion}

A significant difference between the basic EPEA parameters between the main and control groups was found (Table).

The energy level is a key integral indicator of the EPEA method. It demonstrates the intensity of intermolecular transport of excited electrons and the migration of energy of electrical excitation of living tissues [4-6, 8]. The energy functional state of tissues of all respondents in the control group and in 59(97\%) respondents of group 1 and in $56(97 \%)$ respondents of group 2 was in the range of optimal energy levels. However, the indicators of patients with NCDs corresponded to the lower limit of the established norm and they were significantly lower than the corresponding indicators in the control group. This indicates different levels of metabolic processes in the tissues of healthy people and patients with NCDs. There was no significant difference between the corresponding indicators in patients of groups 1 and 2. The trend of quantitative proximity of the relevant indicators was observed when compared. This can be interpreted as a fundamentally identical nature of the metabolic processes in the tissues of patients with different forms of coronary heart disease, which creates a corresponding characteristic level of energy supply and reflection in the EPEA (Table).

EPEA reflects the intensity of electromagnetic components of chemical reactions in accordance with the number and nature of the release/emission of photons at all levels of the morphological hierarchy (from subatomic to organismic). These metabolic processes are influenced by metabolic regulation, so EPEA values are more stable in contrast to, for example, HRV values, which change rapidly during the orthostatic test due to autonomic regulation [4-7]. This was noted in previous works [8] and this is confirmed by the absence of significant changes in the relevant indicators after performing an orthostatic test (Table).

Chronic stress affects the course of chemical reactions and the nature of photon emission by changes in metabolic regulation, so the level of stress can be objectively determined by calculating the EPEA [1, 4-6]. Moderate stress and anxiety were found in all groups in the majority of respondents: they were in $13(21 \%)$ and $41(67 \%)$ respondents groups 1 ; they were in $12(21 \%)$ and $41(71 \%)$ respondents in groups 2 ; they were in 16 $(29 \%)$ and $32(57 \%)$ respondents of the control group. 
Comparative characteristics of the parameters of the EPEA

\begin{tabular}{|c|c|c|c|c|c|c|c|c|c|c|}
\hline \multirow{2}{*}{ Parameter } & \multicolumn{2}{|c|}{ Control group $(n=56)$} & \multicolumn{4}{|c|}{ Group1 (n=61) } & \multicolumn{4}{|c|}{ Group2 $(n=58)$} \\
\hline & $\begin{array}{l}\text { background } \\
\text { state }\end{array}$ & $\begin{array}{c}\text { orthostatic } \\
\text { test }\end{array}$ & $\begin{array}{l}\text { background } \\
\text { state }\end{array}$ & value & $\begin{array}{c}\text { orthostatic } \\
\text { test }\end{array}$ & value & $\begin{array}{l}\text { background } \\
\text { state }\end{array}$ & $P$ value & orthostatic test & value \\
\hline Stress, c.u. & $3.68 \pm 0.70$ & $3.63 \pm 0.86$ & $3.77 \pm 0.93$ & 0.7 & $3.49 \pm 0.66$ & 0.38 & $3.76 \pm 0.79$ & 0.7 & $3.57 \pm 0.62$ & 0.7 \\
\hline$E^{*} 10^{-2}, J$ & $52.49 \pm 3.57$ & $53.41 \pm 3.51$ & $48.08 \pm 5.17$ & $<0.0001$ & $50.43 \pm 5.62^{*}$ & 0.0007 & $47.58 \pm 4.39$ & $<0.0001$ & $47.92 \pm 4,70^{*}$ & $<0.0001$ \\
\hline B, \% & $97.15 \pm 2.02$ & 2.23 & 4 & 1 & 82 & 0476 & 18 & 0424 & 32 & 0.2476 \\
\hline $\mathrm{BL}, \%$ & $91.74 \pm 6.32$ & $92.92 \pm 5.94$ & $83.94 \pm 9.50$ & $<0.0001$ & $86.44 \pm 8.67$ & $<0.0001$ & $84.68 \pm 9.05$ & $<0.0001$ & $85.25 \pm 9.0$ & $<0.0001$ \\
\hline $\mathrm{BR}, \%$ & $91.91 \pm 6$ & $9 \pm 5.08$ & $87.96 \pm 7.83$ & 0.014 & $87.63 \pm 8.37$ & $<0.0001$ & $86.26 \pm 7.15$ & 0.0002 & 3.57 & 0.0017 \\
\hline FC & & & & & & & & & & 0.8 \\
\hline EC & & & & 001 & & 0.0001 & 1 & 22 & & $<0.0001$ \\
\hline$S_{E F} L$ & $887 \pm 6356$ & 73784 & $61988 \pm 6644$ & \begin{tabular}{|c|}
$<0.001$ \\
\end{tabular} & $64638 \pm 6810$ & $<0.0001$ & $62075 \pm 6641$ & $<0.0001$ & $63550 \pm 5304$ & $<0.0001$ \\
\hline$E_{E F L} L, J$ & $33.57 \pm 4.12$ & $33.8 \pm 3.72$ & $25.13 \pm 4.0$ & \begin{tabular}{|c|}
$<0.0001$ \\
\end{tabular} & $24.84 \pm 3.98$ & $<0.0001$ & $26.96 \pm 4.34$ & $<0.0001$ & $25.64 \pm 3.55$ & $<0.0001$ \\
\hline$S_{E F} F$, c.u. & $70100 \pm 5564$ & $71675 \pm 5534$ & $62420 \pm 7281$ & $<0.0001$ & $64656 \pm 6913$ & $<0.0001$ & $64212 \pm 7308$ & $<0.0001$ & $64970 \pm 5982$ & $<0.0001$ \\
\hline$E_{E F} F, J$ & $31.69 \pm 3.66$ & $32.83 \pm 3.71$ & $25.29 \pm 4.34$ & \begin{tabular}{|c|}
$<0.0001$ \\
\end{tabular} & $26.89 \pm 4.38$ & $<0.0001$ & $25.97 \pm 4.27$ & $<0.0001$ & $26.34 \pm 3.81$ & $<0.0001$ \\
\hline$S_{E F} R, c . u$ & $72189 \pm 6176$ & $73759 \pm 5900$ & $62359 \pm 7842$ & $<0.0001$ & $65166 \pm 7165$ & $<0.0001$ & $63638 \pm 7734$ & $<0.0001$ & $64670 \pm 6760$ & $<0.0001$ \\
\hline$E_{E F} R, J$ & $32.72 \pm 3.89$ & $33.74 \pm 3.85$ & $25.28 \pm 4.60$ & \begin{tabular}{|l|}
$<0.0001$ \\
\end{tabular} & $27.11 \pm 4.43$ & $<0.0001$ & $25.81 \pm 4.59$ & $<0.0001$ & $26.31 \pm 4.26$ & $<0.0001$ \\
\hline
\end{tabular}

Note * - the difference Mann-Whitney test is reliable at $p<0.05$ between the characteristics Group1 and Group2; $P$ value - the difference Mann-Whitney test between the characteristics of the study groups and the control group; clinical interpretation of parameters: Stress - 0-2 c.u. - its calm state, 2-3 - its optimal condition, 3-4 c.u. - its anxiety, 4-6 c.u. - its average condition, 6-8 c.u. - its increased stress levels, 8-10 - its distress; E -0-20 J - its low level, 20-40 J - its low level, 40-70 J - its optimal level, 70$90 \mathrm{~J}$ - its increased level, $90-100 \mathrm{~J}$ - its high level; B - 0-50\% - its very low balance; 50-90\% - its low balance; $90-100 \%$ - its optimal balance; $B L, \%$ 0-5\% - its optimal balance; 5-10\% - its average imbalance; $10 \%$ - >15\% - its severe imbalance; $B R, \%$ - 0-5\% - its optimal balance; $5-10 \%$ - its average imbalance 10\% -> 15\% - its severe imbalance.

Energy balance indicators are important for clinical interpretation. A significant difference between the photon emission rates on the left and right can indicate disorders in the functioning of the organs and this can be a topical reference point of pathology. The phenomenon of significant energy asymmetry (lateralization syndrome) is a manifestation of dysfunction of the autonomic nervous system and it is an objective sign of reduced reserves of adaptation, a disorder of energy homeokinesis [1, 4-6]. Signs of the lateralization phenomenon were diagnosed in $13(21 \%)$ patients of group 1 and $14(24 \%)$ patients of group 2. Optimal balance levels were observed in the control group.

\section{Conclusions}

Patients with NCDs - coronary heart disease have a lower level of intensity of electromagnetic components of chemical reactions of metabolism in contrast to healthy individuals, objective signs of autonomic imbalance, reduced adaptation, increased stress levels. The EPEA method is a modern high-tech available valid instrumental technique. The EPEA method can provide an opportunity to objectively assess the course of energy processes of tissue metabolism, to determine the level of stress, the parameters of the balance of the body. The EPEA method can be recommended as promising for use in clinical practice of objective structured clinical examination of patients.

\section{References}

1. Bio-Well Manual. 2019. [Internet]. Available from: https://www.iumab.org/2019-bio-well-user-manual/
2. Burgos R.C.R., Schoeman J.C., van Winden L.J., Červinková K., Ramautar R., Van Wijk E.P.A., Cifra M., Berger R., Hankemeier T., van der Greef J. Ultra-weak photon emission as a dynamic tool for monitoring oxidative stress metabolism. Scientific RepoRts, 2017; 1-9 doi: 10.1038/s41598-017-01229-x

3. esmaeilpour t., fereydouni e., Dehghani f., Bókkon i., panjehshahin M.-R., császár-nagy n., Ranjbar M., Salari $\mathrm{V}$. An experimental investigation of Ultraweak photon emission from Adult Murine neural Stem cells. Scientific RepoRts, 2020; 10:453. doi:10.1038/s41598-019-57352-4

4. Korotkov K. Energy Fields Electrophotonic Analysis in Humans and Nature: Energy Fields Analysis. San Petersburg; 2014, 237 p.

5. Korotkov K. Review of EPI papers on medicine and psychophysiology published in 2008-2018. Int JComplement Alt Med., 2018;11(5):311-315. doi: 10.15406/ijcam.2018.11.00417

6. Korotkov K.G. Principles of the human body functioning and their applications in integrative medicine (review). J Appl Biotechnol Bioeng., 2018;5(6):346-348. doi:10.15406/jabb.2018.05.00163

7. Mintser O.P, Potiazhenko M.M., Nevoit G.V. Evaluation of the human bioelectromagnetic field in medicine: the development of methodology and prospects are at the present scientific stage. Wiadomości Lekarskie, 2019;5, II: 1117-1121. doi:10.36740NLek202006139

8. Nevoit G.V., Potiazhenko M.M., Mintser O.P., Babintseva L.Yu. Electro-Photonic Emission Analysis and HardwareSoftware recoding of heart Rate Variability during an Objective Structured Clinical Examination. The world of medicine and biology, 2020;4:107-111 doi: 10.26724/20798334-2020-4-74-107-111

Матеріал надійшов до редакції 17.03.2021 